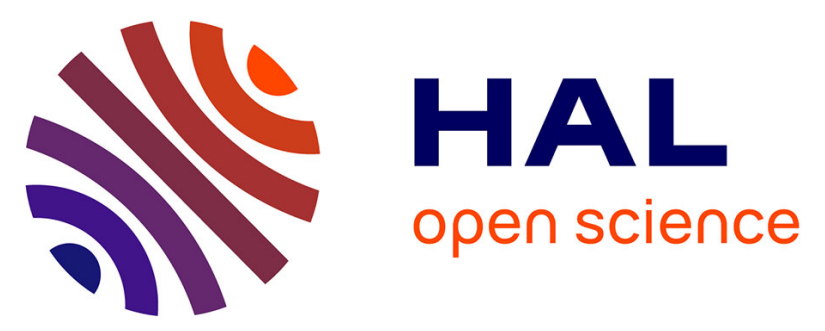

\title{
Sequential double extension twinning in a magnesium alloy: Combined statistical and micromechanical analyses
}

Zhang-Zhi Shi, Yudong Zhang, Francis Wagner, Thiebaud Richeton, Pierre-Alexandre Juan, Jean-Sebastien Lecomte, Laurent Capolungo, Stéphane Berbenni

\section{To cite this version:}

Zhang-Zhi Shi, Yudong Zhang, Francis Wagner, Thiebaud Richeton, Pierre-Alexandre Juan, et al.. Sequential double extension twinning in a magnesium alloy: Combined statistical and micromechanical analyses. Acta Materialia, 2015, 96, pp.333-343. 10.1016/j.actamat.2015.06.029 . hal-01514963

\section{HAL Id: hal-01514963 \\ https://hal.univ-lorraine.fr/hal-01514963}

Submitted on 17 Dec 2019

HAL is a multi-disciplinary open access archive for the deposit and dissemination of scientific research documents, whether they are published or not. The documents may come from teaching and research institutions in France or abroad, or from public or private research centers.
L'archive ouverte pluridisciplinaire HAL, est destinée au dépôt et à la diffusion de documents scientifiques de niveau recherche, publiés ou non, émanant des établissements d'enseignement et de recherche français ou étrangers, des laboratoires publics ou privés. 


\title{
Sequential double extension twinning in a magnesium alloy: Combined statistical and micromechanical analyses
}

\author{
Zhang-Zhi Shi ${ }^{\mathrm{a}, *}$, Yudong Zhang ${ }^{\mathrm{a}}$, Francis Wagner ${ }^{\mathrm{a}}$, Thiebaud Richeton ${ }^{\mathrm{a}}$, Pierre-Alexandre Juan ${ }^{\mathrm{b}, \mathrm{a}}$, \\ Jean-Sébastien Lecomte ${ }^{a}$, Laurent Capolungo ${ }^{b}$, Stéphane Berbenni ${ }^{a}$ \\ a Laboratoire d'Etude des Microstructures et de Mécanique des Matériaux, LEM3, CNRS 7239, Université de Lorraine U.L. - Metz, Ile du Saulcy, 57045 Metz, France \\ ${ }^{\mathrm{b}}$ George Woodruff School of Mechanical Engineering, Georgia Institute of Technology, UMI 2958 Georgia Tech-CNRS, 57070 Metz, France
}

\section{A R T I C L E I N F O}

\section{Keywords:}

Magnesium alloy

EBSD

Twinning

Micromechanics

\begin{abstract}
A B S T R A C T
A statistical analysis is conducted on sequential $\{10-12\}-\{10-12\}$ double extension twinning in a Mg AZ31 alloy obtained after two mutually perpendicular successive compressions. Indeed, primary twins form during the first compression, which produce secondary twins during the second compression. Here, "sequential double twinning" is adopted to describe this twinning mechanism, which is different from classic double twinning obtained during monotonic loading. Double extension twins, no matter sequential or classic ones, can be classified into Groups I-IV according to their misorientations with respect to the parent grain. In the present study, the majority of the sequential double extension twins have relatively high Schmid factors (SFs) and a misorientation of $\langle 0,14,-14,1\rangle 60^{\circ}$ with respect to the parent grain (Group III). However, the classical SF based analysis fails to explain why secondary twin variants in Group III are preferred over their nearest orientated neighbors in Group IV, since their SF differences are negligible. Therefore, a static double inclusion model is developed to analytically compute the elastic energy in a final configuration of sequential double twinning. The model well explains the predominance of secondary twins in Group III by a noticeable greater decrease of free energy after secondary twinning.
\end{abstract}

\section{Introduction}

Deformation twinning influences the mechanical responses of Mg and its alloys, especially at low temperatures, through the local strain and crystallographic reorientations associated with it [1]. Primary $\{10-12\}<-1011>$ extension twins (ETWs) result from a shear of $\gamma=0.129$ (when $c / a=1.624$ ) and cause an extension of the hexagonal lattice along the $c$-axis, and a rotation of the crystal by $86.3^{\circ}$ about a $<1-210>$ direction [2]. Due to the reorientation of the crystal within the primary ETW, deformation modes that were not active prior to twinning may be activated. Wrought $\mathrm{Mg}$ alloys often have initial textures with the c-axes of most grains roughly parallel to the normal direction for rolling or perpendicular to the extrusion direction for extrusion [3,4], which is prone to form ETWs under compression along the processing direction. For wrought AZ31 alloy (Mg-3Al-1Zn), secondary ETWs seldom form in the primary ETWs during a uni-directional deformation path at room temperature and above $[3,4]$. This can be understood

\footnotetext{
* Corresponding author.

E-mail addresses: ryansterne@sina.com, ryansterne@gmail.com (Z.-Z. Shi).
}

based on two arguments. First, slip is easier to be activated than twinning in the primary ETWs. The other is the directional nature of deformation twinning, which results in a twin texture with the c-axis of the twinned region aligned around the compressive loading direction, and thus unfavorable for the secondary ETWs [4,5]. In contrast, secondary ETWs can easily form in primary $\{10-11\}<10-1-2>$ contraction twins (CTWs) during uni-directional deformations [6-9]. Studies related to the CTW-ETW double twinning mechanism have been widely reported [6-9]. However, the ETW-ETW double twinning is much less studied.

Recently, a few cases of ETW-ETW double twinning have been observed in AZ80 Mg alloy (Mg-8Al-0.5Zn) uni-directionally compressed at $77 \mathrm{~K}[10]$. The ETW-ETW double twinning at such a low temperature can be attributed to the more athermal nature of the critical resolved shear stress (CRSS) for deformation twinning compared with the thermally activated nature of the CRSSs for non-basal slip systems that increases strongly with decreasing temperatures [11]. At ambient and elevated temperatures, rare cases of ETW-ETW double twinning have also been detected in direct extruded $\mathrm{Mg}-0.3$ at.\% $\mathrm{Al}$ alloy, which were claimed to result 
from multi-axial stress fields [12]. However, ETW-ETW double twinning is more frequently observed in hot rolled AZ31 alloy after pre-compression along the rolling direction (RD) and further compression along the transverse direction (TD) at ambient temperature, and vice versa [13-15]. Following pre-compression along the $\mathrm{RD}$, the initial and the twin textures coexist in the material, with the $c$-axes of crystals almost parallel to the normal direction (ND) and the RD, respectively [13]. The further compression along the TD enables three twinning mechanisms: primary ETW in residual grains, secondary ETW in primary ETW, and detwinning of primary ETW [13]. It was recently claimed that the selection of secondary ETW variant is governed by Schmid factors (SFs), except a few twins with low SFs claimed to be initiated by the increased local stress at primary ETW-ETW intersection boundaries [14].

Since metal forming processes, such as bending and drawing, introduce multiple compressive stresses, the study of double twinning generated under different compressive loadings is helpful for a better control of microstructures and related mechanical properties of wrought materials. The influence of the primary ETW on the selection of secondary ETW variant requires more investigation. It is unclear how the formation of a secondary ETW changes the mechanical fields in the parent primary ETW. First, this paper conducts a statistical analysis of sequential ETW-ETW double twins in an AZ31 alloy subjected to two successive orthogonal compressive loadings. Then, a double inclusion model is developed in order to analytically compute the elastic energy in the case of sequential double twinning observed during the second loading stage. The term "sequential" differs from classic double twinning due to monotonic loading. This micromechanical scheme describes a double inclusion topology with "eigenstrains" embedded in an elasto-plastic matrix. One ellipsoidal inclusion is taken as the primary ETW, which contains the other ellipsoidal inclusion as the secondary ETW.

\section{Experimental procedure}

The material used is a conventional hot-rolled commercial AZ31 alloy (Mg-3Al-1Zn in wt.\%) sheet of $15 \mathrm{~mm}$ thickness, which was annealed at $400{ }^{\circ} \mathrm{C}$ for $2 \mathrm{~h}$ after rolling. Cube specimens with a length of $10 \mathrm{~mm}$ were cut from the sheet. The rolling, transverse and normal directions of the rolled sheet are designated as RD, TD and ND, respectively. Compression tests were performed at room temperature with a strain rate of $1 \times 10^{-3} / \mathrm{s}$. The cubes were compressed in three ways: (i) only along the RD (uni-RD); (ii) only along the TD (uni-TD); (iii) first along the RD, unloaded and then along the TD (RD + TD). The crystallographic textures of the samples were examined by X-ray diffraction (XRD) pole figure analysis, using a three circles diffractometer with a curved detector (INEL CPS120). The XRD pole figures presented in this paper are recalculated from the orientation distribution function (ODF), which is obtained from the original measured pole figures with the MATLAB $^{\mathrm{TM}}$ toolbox MTEX [16].

After $1.8 \% \mathrm{RD}+1.3 \% \mathrm{TD}$ compressions, the specimen were sectioned in a direction normal to the ND (i.e., ND sectioned samples) or the RD (i.e., RD sectioned samples) for microstructural analysis within the bulk. The experimental results obtained from both kinds of sectioned samples were extracted for a statistical analysis. No metallurgical difference rises from the sectioning method. Only the microstructures related to the ND sectioned samples are reported in the present paper. The sectioned faces were ground using $\mathrm{SiC}$ papers with grits from 2400 to 4000 and then electrolytically polished in an electrolyte of $62.5 \%$ phosphoric acid and $37.5 \%$ ethanol at $3 \mathrm{~V}$ for $30 \mathrm{~s}$ and then at $1.5 \mathrm{~V}$ for $2 \mathrm{~min}$, at $15^{\circ} \mathrm{C}$. The electron backscatter diffraction (EBSD) measurements were performed using a JEOL 6500F FEG SEM microscope with Channel 5 analysis system (Oxford HKL) [17]. The step size adopted for EBSD measurements was $0.3 \mu \mathrm{m}$.

\section{Results}

\subsection{Mechanical response and microstructure}

The as-received material is recrystallized without twins and the $c$-axes of grains center around the ND (i.e., the initial texture), according to a previous study [18] (or see later in Fig. 2a). The engineering stress-strain curves of uni-TD and RD + TD cubes are illustrated in Fig. 1a. A yield plateau is clearly seen from both the curves of the uni-TD and the first RD compressions, which is a typical signature of $\{10-12\}$ extension twinning on the macroscopic stress response [4]. Since compression along the RD or along the TD exerts extension along the c-axes of the grains according to the

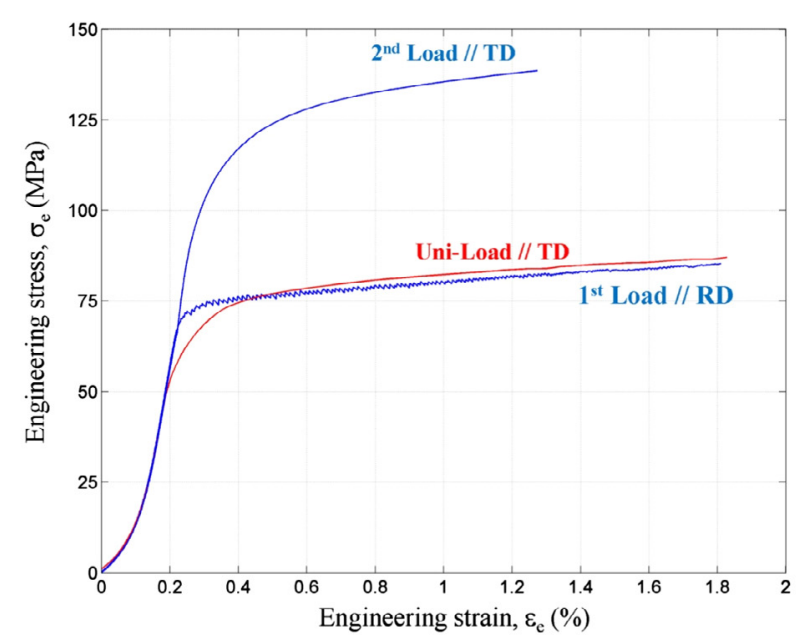

(a)

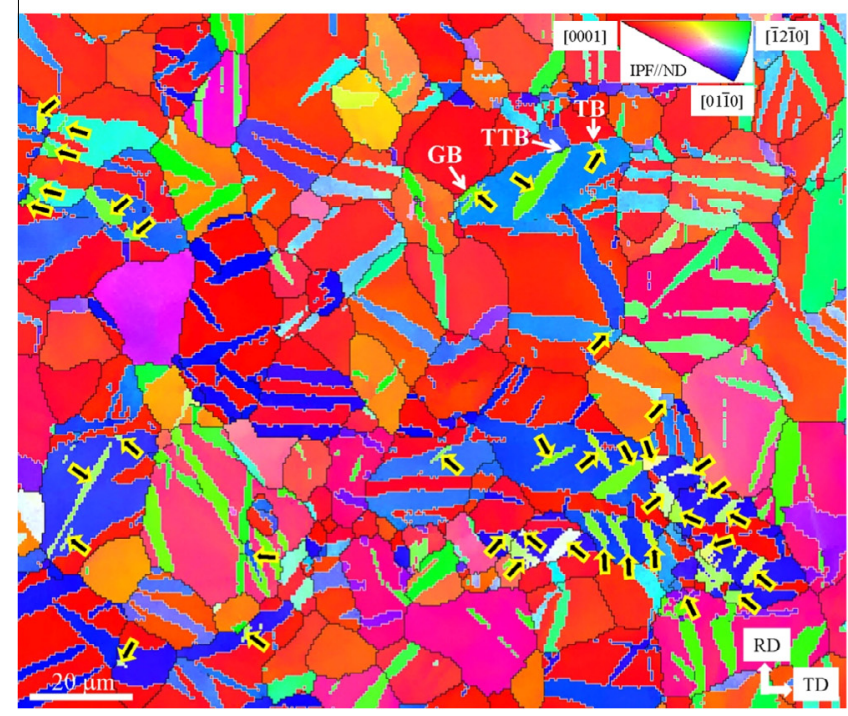

(b)

Fig. 1. (a) Engineering stress-strain curves of RD + TD and uni-TD compressed cubes. (b) A representative EBSD micrograph of the sample after 1.8\% RD + 1.3\% TD compressions, including the ND inverse pole figure (IPF) color code. Boundaries in black separate two regions with misorientations $>5^{\circ}$, while those in white are special ones with an misorientation of $\left\langle-12-10>86^{\circ}\right.$ (offset $\pm 5^{\circ}$ ). The arrows point out 39 secondary ETWs. GB, TB and TTB refer to grain boundary, twin boundary and twin-twin boundary, respectively. The same coloring code is adopted for all the figures afterwards. (For interpretation of the references to color in this figure legend, the reader is referred to the web version of this article.) 


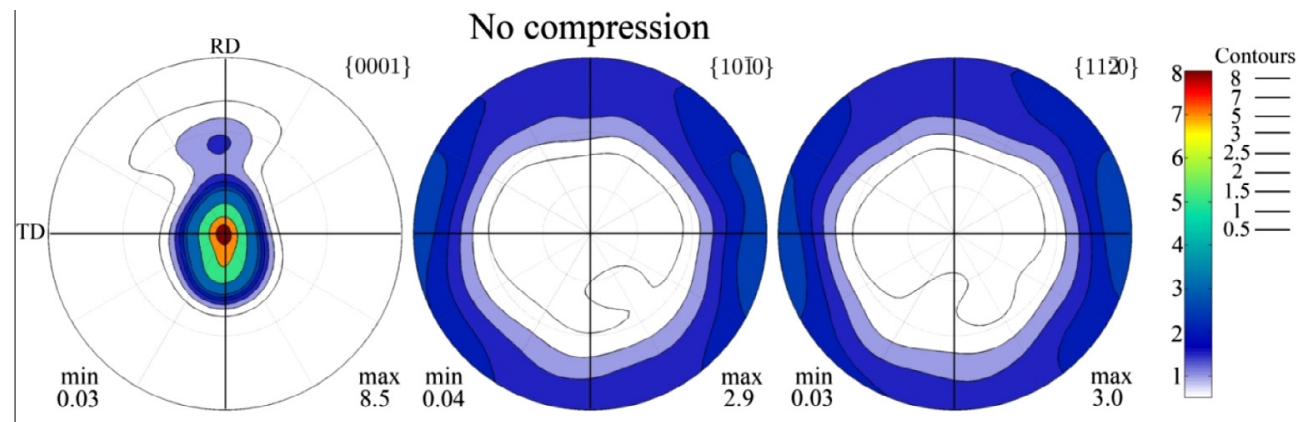

(a)

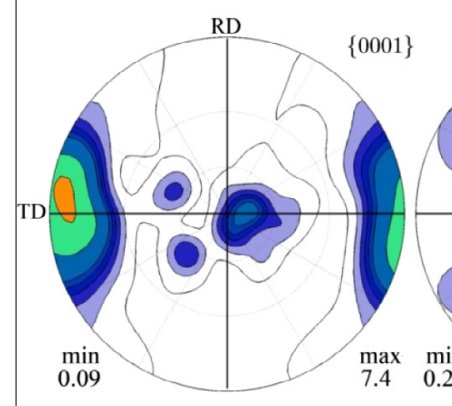

TD compression

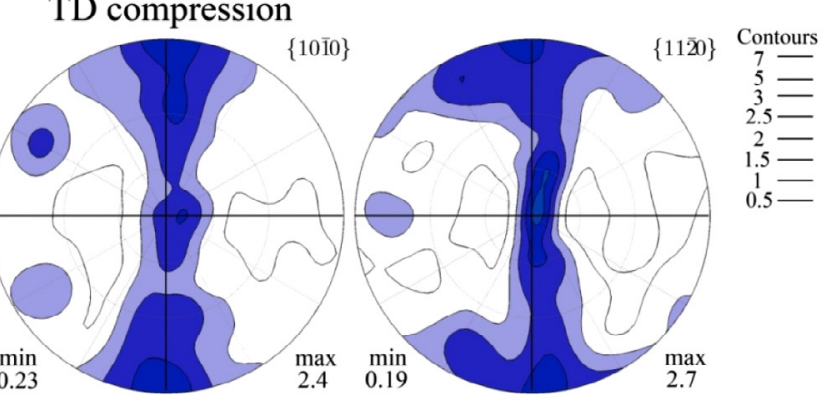

(b)
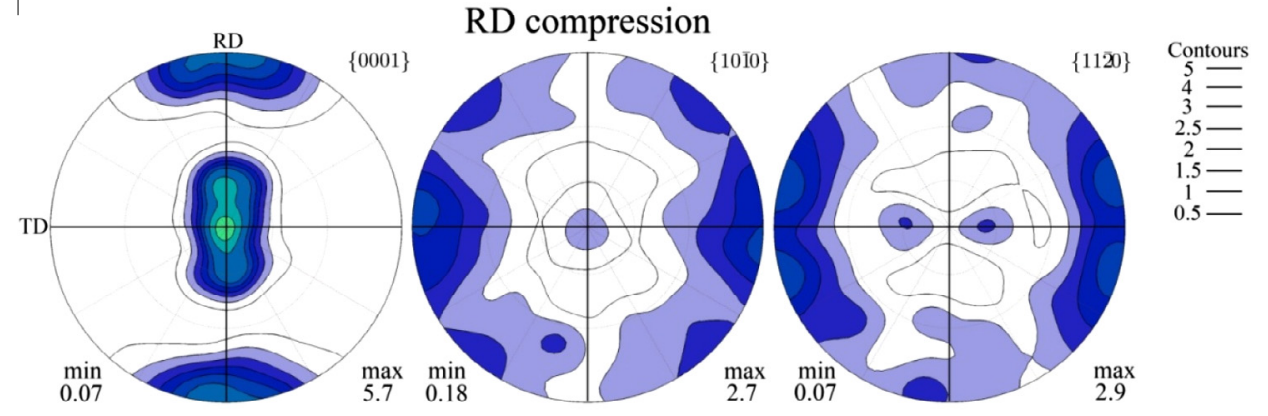

(c)

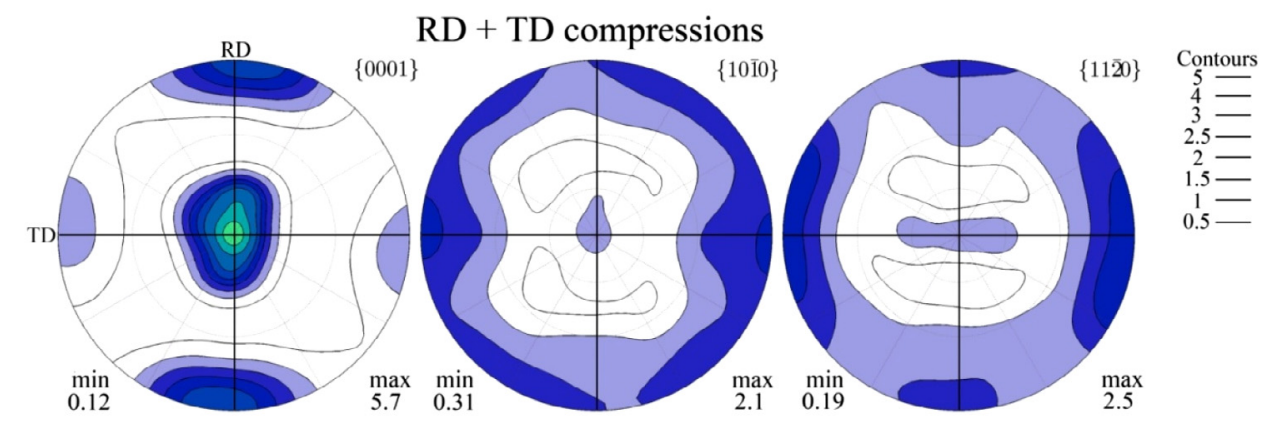

(d)

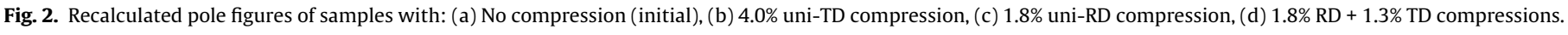

initial texture, $\{10-12\}$ extension twinning with low CRSS [19] can easily be activated. The yield stresses (at $0.2 \%$ strain) of the first $\mathrm{RD}$ and the uni-TD compressions are $\sim 75 \mathrm{MPa}$ and $\sim 72 \mathrm{MPa}$, respectively. It is also found by other studies [4,20] that uni-RD and uni-TD compressions of rolled AZ31 alloy samples are both featured with yield plateaus and comparable yield stresses. Such a yield plateau is shifted up in the curve of the second TD compression, which is even clearer in [21] with compressive strains going as far as failure. The shifted yield plateau indicates an increase of twinning CRSS due to a grain refining effect caused by the primary twinning. Such a size effect was attributed to a Hall-Petch type's relationship in [13] where the same sequential loading scenario was investigated (i.e., RD + TD compressions). During the second TD compression, the yield stress increases to $\sim 120 \mathrm{MPa}$ and the strain hardening rate after yielding is $\sim 2.8$ times that of the uni-TD compression. Therefore, it is reasonably assumed that the additional interfaces generated by primary twinning in the first RD compression may act as strong barriers for slip dislocations 
and twinning during the second TD compression. Such constrained plasticity may be responsible for an increase of the yield strength.

EBSD measurements were conducted on the sample after $1.8 \%$ $\mathrm{RD}+1.3 \% \mathrm{TD}$ compressions, together covering a large region of $0.82 \mathrm{~mm}^{2}$ containing 4481 grains, without accounting for those intercepted by EBSD map borders (see Table 1). A representative microstructure is shown in Fig. 1b with an EBSD map based on ND inverse pole figure. Boundaries with misorientations larger than $5^{\circ}$ are outlined in black, while those with misorientations of $<-12-10>86^{\circ}$, with a tolerance of $\pm 5^{\circ}$ deviation from the axis and the angle, are delineated in white. Lamellar-shaped regions contoured by white boundaries correspond to primary ETWs in the grain matrices or to secondary ETWs in the primary ETWs. In Fig. $1 \mathrm{~b}$, secondary ETWs are pointed out by arrows. As shown in Table 1, the detected 11,052 primary ETWs take up $27.94 \%$ of the total area. In average, there are 2-3 primary ETWs in a grain. By contrast, the detected 585 secondary ETWs take up only $0.42 \%$ of the total area.

The majority of the secondary ETWs are connected with boundaries, including grain boundary (GB), twin boundary (TB) between a primary twin and the grain matrix, and twin-twin boundary (TTB) between two primary twins in the same grain. Typical examples are pointed out in Fig. 1b. Several secondary ETWs can form in one primary ETW, e.g. the three secondary ETWs close to the marks of GB, TB and TTB in the top right of Fig. 1b. No secondary ETWs are detected in specimen after uni-RD compressions, which is consistent with other studies $[4,18]$. Therefore, it is concluded that the secondary ETWs observed in Fig. $1 \mathrm{~b}$ form during the second TD compression.

The recalculated $\{0001\},\{01-10\}$ and $\{11-20\}$ pole figures of the cubes without compression, with $4.0 \%$ uni-TD compression, with $1.8 \%$ uni-RD compression, and with $1.8 \% \mathrm{RD}+1.3 \%$ TD compressions are shown in Fig. 2a-d, respectively. Fig. 2a-c shows that the $\{0001\}$ poles of grains turn from directions near the ND to those near the compressive loading directions, i.e., the TD (for uni-TD compression) or the RD (for uni-RD compression), which gives rise to a notable twin texture component due to primary twinning. This indicates that the $c$-axes of the majority of the primary ETWs and the residual grains after the first RD compression are both subjected to extension in the second TD compression, which will lead to the formation of secondary ETWs and primary ETWs, respectively. Fig. 2d shows that the primary ETWs formed in the second TD compression rotate the $c$-axes of the grains to the TD. Although the small volume fraction of secondary ETWs that can be inferred from Table 1 implies that their influence on texture will be much lower than that of the primary ETWs, the microstructure characterized by EBSD (Fig. 1b) reveals their existence. Hereafter, the sequential ETW-ETW double twins will be intensively investigated.

\subsection{Geometry of double extension twins}

No matter sequential or classic double extension twins, they have the same geometry. Thus, the general terminology of double extension twin is used in this section and in situations alike. There are 12 rotational symmetry operations of hcp crystals, leading to 6

\section{Table 1}

Numbers (No.), areas, area fractions and the average diameters $(D)$ of EBSD measured grains and twins in a sample after $1.8 \% \mathrm{RD}+1.3 \% \mathrm{TD}$ compressions.

\begin{tabular}{lllll}
\hline & No. & Area $\left(\times 10^{3} \mu \mathrm{m}^{2}\right)$ & Area fraction $(\%)$ & $D(\mu \mathrm{m})$ \\
\hline Grains & 4481 & 823 & 100 & 16.34 \\
Primary ETWs & 11,052 & 230 & 27.94 & 5.24 \\
Secondary ETWs & 585 & 3.47 & 0.42 & 1.37 \\
\hline
\end{tabular}

primary ETW variants. The misorientations between the 6 primary ETW variants can be expressed by three axis-minimum angle pairs [2]: $\langle 1-210\rangle 7.4^{\circ},\langle 01-10\rangle$ (accurately $\langle 0,14,-14,1\rangle$ ) $60^{\circ}$, and $<17-80>\left(60.4^{\circ}\right)$. In the case of double extension twinning, a given primary ETW has 6 possible secondary ETW variants so that there are 36 possible double ETW variants in total. For convenience, the 6 possible ETW variants, no matter a primary one (PV) or a secondary one (SV), are numbered as follows: (1) $\{10-12\}<-1011>$, (2) $\{01-12\}<0-111>$, (3) $\{-1102\}<1-101>$, (4) $\{-1012\}<$ $10-11>$, (5) $\{0-112\}<01-11>$, (6) $\{1-102\}<-1101>$. The misorientation between a ETW-ETW double twin and the grain matrix can be determined by applying two successive rotations of $180^{\circ}$ around the respective normal of the primary and the secondary twinning planes. Table 2 lists all the 36 misorientations given by axis-minimum angle pairs. According to the table, the 36 double ETW variants can be divided into 4 misorientation groups according to their misorientations with respect to the grain matrix, as listed in Table 3. Groups I and II each contain 6 variants, while Groups III and IV each contain 12 variants. Variants in Group I have no misorientation with respect to the grain matrix so that it is equivalent to detwinning.

Conveniently, Fig. 3a gives the reorientations of the $\{0001\}$ poles after double extension twinning. The grain matrix is ideally assumed to have Euler angles of $\left(0^{\circ}, 0^{\circ}, 0^{\circ}\right)$ in the sample frame defined by $\mathbf{x} / / \mathrm{RD}, \mathbf{y} / / \mathrm{TD}$ and $\mathbf{z} / / \mathrm{ND}$. The 6 possible secondary ETW variants corresponding to a primary ETW are characterized by the four misorientation groups as listed in Table 3 . The $\{0001\}$ pole of the variant in Group I coincides with that of the grain matrix, which is equivalent to detwinning. Fig. 3b shows the reorientations of $\{10-12\}$ twinning planes after double extension twinning. Since a twinning plane is an invariant plane, shared by a twin and its parent crystal, such a plane between the primary twin and its matrix grain is marked by an open square in the figure. The 6 possible $\{10-12\}$ secondary twinning planes are also characterized by the four misorientation groups (Table 3 ). The angles between the primary twinning plane and its 6 secondary twinning planes are given in Fig. $3 \mathrm{~b}$. The angle associated with the variant in Group I is $0^{\circ}$ because its twinning plane coincides with that of the primary twin. Fig. $3 c$ shows the reorientations of $<-1011>$ twinning directions after double extension twinning.

\subsection{Schmid factor analysis of secondary ETWs}

Twinning may produce lattice distortion in their vicinities [6,7], which will deviate the measured orientation of a twin from its ideal orientation calculated according to the orientation of its matrix. Therefore, uncertainty may arise in the identification of twin variant. In the present study, a detected twin deviates less than $7^{\circ}$ with respect to the ideal orientation of a possible twin variant. For a detected twin, the variant that matches closest is marked as M1, while the variant that matches the second closest is marked as M2. When the misorientation angle between M1 and M2 is larger than $3^{\circ}$, a detected twin is identified by M1. In what follows this is referred to as an identifiable twin. In the present study, all secondary ETWs taken into consideration coexist with their parent primary ETWs, which further facilitates to eliminate the ambiguity of secondary twin variants. The above conditions apply to 383 identifiable secondary ETWs (i.e., 65.5\%) of 585 detected ones.

During a compression test, internal stresses can develop in the twinned and the parent lattices in different ways [22-24]. In the present study, the local stress field responsible for activating a secondary ETW should be the combination of the applied TD stress field and the internal stress field. Although it is difficult to accurately evaluate the internal stress field, the applied TD stress field can be used to calculate the "macro" SF of a secondary twin, which will suggest the role of the internal stress field. If the internal stress 
Table 2

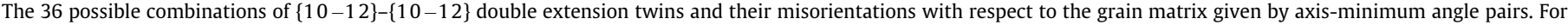

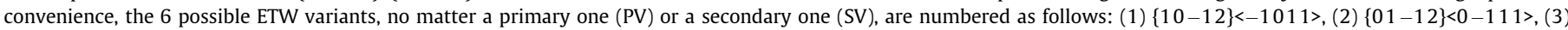
$\{-1102\}<1-101>$, (4) $\{-1012\}<10-11>$, (5) $\{0-112\}<01-11>,(6)\{1-102\}<-1101\rangle$.

\begin{tabular}{|c|c|c|c|c|c|c|}
\hline & SV1 & SV2 & SV3 & SV4 & SV5 & SV6 \\
\hline PV1 & $0^{\circ}$ & $<0,14,-14,1>60^{\circ}$ & $<7-810>60.4^{\circ}$ & $<1-210>7.4^{\circ}$ & $<1-870>60.4^{\circ}$ & $<-14,14,0,-1>60^{\circ}$ \\
\hline PV2 & $<-14,0,14,-1>60^{\circ}$ & $0^{\circ}$ & $<-14,14,0,1>60^{\circ}$ & $<8-1-70>60.4^{\circ}$ & $<2-1-10>7.4^{\circ}$ & $<8-7-10>60.4^{\circ}$ \\
\hline PV3 & $<71-80>60.4^{\circ}$ & $<0,-14,14,-1>60^{\circ}$ & $0^{\circ}$ & $<-14,0,14,1>60^{\circ}$ & $<17-80>60.4^{\circ}$ & $<11-20>7.4^{\circ}$ \\
\hline PV4 & $<-12-10>7.4^{\circ}$ & $<-18-70>60.4^{\circ}$ & $<14,-14,0,-1>60^{\circ}$ & $0^{\circ}$ & $<0,-14,14,1>60^{\circ}$ & $<-78-10>60.4^{\circ}$ \\
\hline PV5 & $<-8170>60.4^{\circ}$ & $<-2110>7.4^{\circ}$ & $<-8710>60.4^{\circ}$ & $<14,0,-14,-1>60^{\circ}$ & $0^{\circ}$ & $<14,-14,0,1>60^{\circ}$ \\
\hline PV6 & $<14,0,-14,1>60^{\circ}$ & $<-1-780>60.4^{\circ}$ & $<-1-120>7.4^{\circ}$ & $<-7-180>60.4^{\circ}$ & $<0,14,-14,-1>60^{\circ}$ & $0^{\circ}$ \\
\hline
\end{tabular}

Table 3

The 36 possible double ETW variants classified into 4 misorientation groups according to their misorientations with respect to the grain matrix.

\begin{tabular}{llc}
\hline Group & Axis-minimum angle pair & Variants \\
\hline I & $0^{\circ}$ (detwinning) & 6 \\
II & $<1-210>7.4^{\circ}$ & 6 \\
III & $<0,14,-14,1>^{*} 60^{\circ}$ & 12 \\
IV & $<17-80>60.4^{\circ}$ & 12 \\
\hline
\end{tabular}

$3.8^{\circ}$ off $\left\langle 01-10>\right.$ and $7.6^{\circ}$ off $\langle 17-80\rangle$.

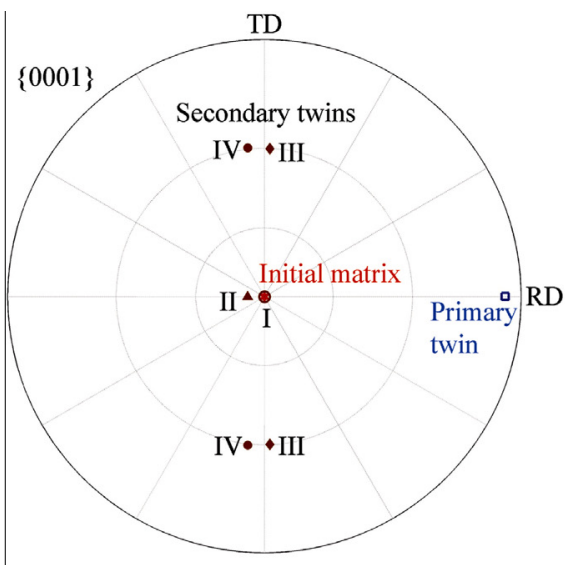

(a) field deviates the local stress field far away from the applied TD stress field, the macro SF of a secondary twin could be low.

Fig. 4a shows the frequency of the 383 identifiable secondary ETWs in terms of their macro (or classic) SFs. Hereafter, SF refers to the macro SF unless otherwise specified. All the secondary ETWs have positive SFs, $78.1 \%$ of which have SFs $>0.3$. Moreover, their SF ratios are calculated in order to evaluate how the applied TD stress determines the variant selection of the secondary ETWs. The $\mathrm{SF}$ ratio is defined as $\mathrm{SF}_{\text {ratio }}=\mathrm{SF} / \mathrm{SF}_{\text {max }}$, where $\mathrm{SF}$ refers to the $\mathrm{SF}$ of an identifiable secondary ETW and $\mathrm{SF}_{\max }$ refers to the maximum

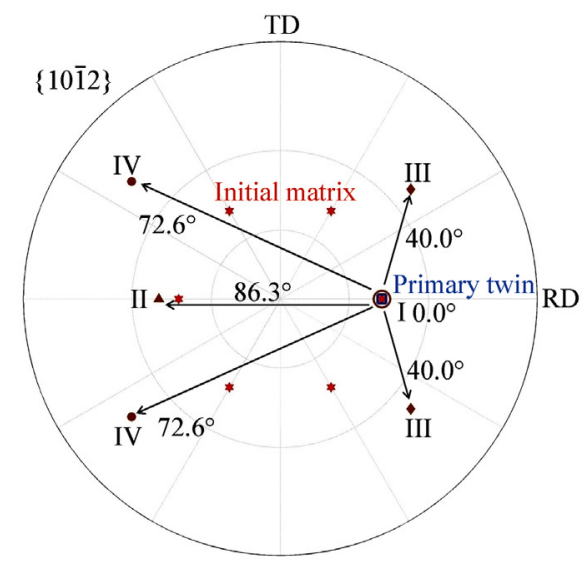

(b)

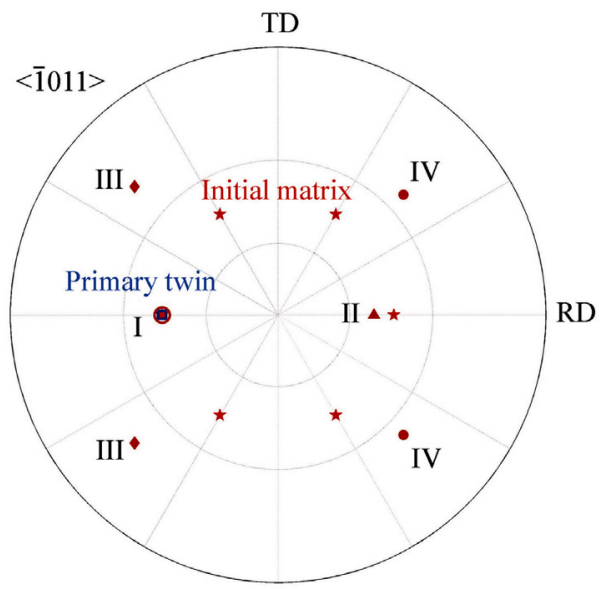

(c)

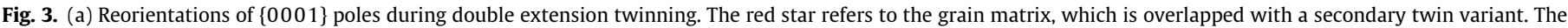

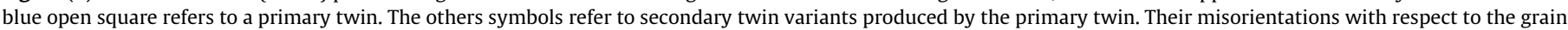

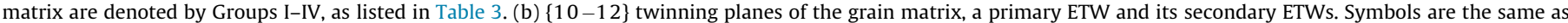

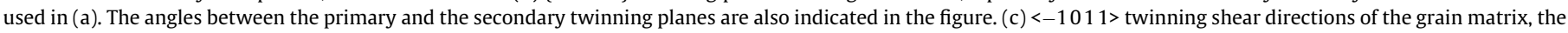
primary ETW in (b) and its secondary ETWs. (For interpretation of the references to color in this figure legend, the reader is referred to the web version of this article.) 


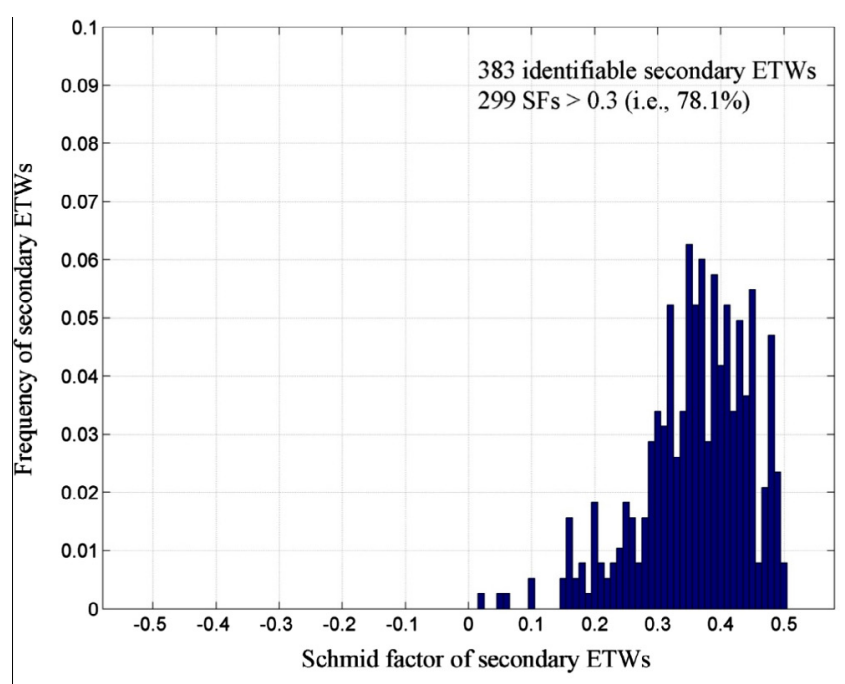

(a)

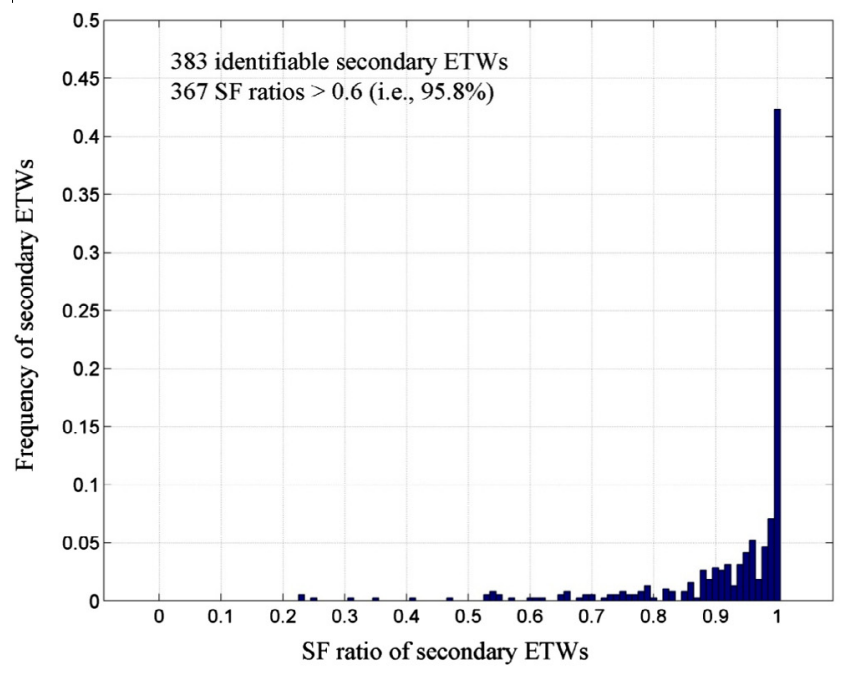

(b)

Fig. 4. For 383 identifiable secondary ETWs: (a) Frequency of the twins in terms of their SFs, (b) frequency of the twins in terms of their SF ratios.

SF among the 6 possible secondary ETW variants of the parent primary ETW. Fig. $4 \mathrm{~b}$ shows the frequency of the 383 identifiable secondary ETWs in terms of their SF ratios. It shows that the large majority (i.e., 95.8\%) of them have $\mathrm{SF}$ ratios $>0.6$, indicating that they correspond to variants with relatively high SFs compared with other possible secondary twin variants in their parent primary twins. A previous study by the present authors [18] on primary ETWs specified twins with either $\mathrm{SF} \leqslant 0$ or $0<\mathrm{SF} \leqslant 0.3$ and $\mathrm{SF}_{\text {ratio }} \leqslant 0.6$ as "low SF" twins. Accordingly, it can be deduced from Fig. 4 that $4.2 \%$ of the secondary ETWs are low SF twins, which is comparable to that of the primary ETWs (i.e., 6.8\% [18]).

The above SF analysis indicates that the applied TD stress plays the most important role for the activation and the selection of the secondary ETW variant after the first $1.8 \%$ RD compression. Considering that the internal stress in the twinned lattice is very low at the beginning of the deformation but dramatically increases with macroscopic strain during a compression test [22], the local stress field could be very different from the applied TD stress field. However, in the present study, internal stresses in the primary ETWs are likely to be much lower than the applied TD stress. The possible reasons are: (i) the first 1.8\% RD compression is not large enough in favor of developing high internal stresses in the primary ETWs; (ii) some probable plastic accommodation mechanisms (slip etc.) can partially relax the internal stresses after the first RD compression; (iii) the yield stress of the second TD compression is $\sim 45 \mathrm{MPa}$ higher than the stress of the yield plateau of the first RD compression (Fig. 1a). Thus, the applied TD stress may be higher than the residual internal stresses.

\subsection{Misorientation preference of secondary ETWs}

As shown in Table 3, the 36 possible double ETW variants can be classified into four misorientation groups. Group I corresponds to detwinning, which is not possible to explore and quantify using such ex-situ observations. Fig. 5a shows the frequency of the 383 identifiable secondary ETWs in terms of their misorientation groups. It clearly indicates that the majority (i.e., 76.0\%) of the twins belong to Group III. The rest belong to Group IV, taking up 24.0\%. None of them belongs to Group II. Fig. 5b shows SFs and SF ratios of the 291 secondary ETWs in Group III. It is seen that $74.2 \%$ of the twins have SFs $>0.3$, while $95.2 \%$ of them have SF ratios $>0.6$. Analogously, SFs and SF ratios of the 92 secondary ETWs in Group IV are given in Fig. 5c, which shows that $90.2 \%$ of the twins have SFs $>0.3$, while $98.9 \%$ of them have SF ratios $>0.6$. Comparatively, it is revealed that the SF rule works in a better way for the secondary ETWs in Group IV.

As seen in Fig. 3a, two nearest neighboring secondary ETW variants with a misorientation of $7.4^{\circ}$ about $\langle 1-210\rangle$ belong separately to Groups III and IV. It can be calculated that the absolute SF difference between them is not larger than 0.05 for any of the twinned grains in the present study, which is too small to be meaningful for twin variant selection. Therefore, the SF analysis cannot explain why secondary ETW variants in Group III are more often preferred over those in Group IV. In the following section, a double inclusion model will be developed to explain it.

\section{Micromechanical analysis for sequential ETW-ETW double twinning}

The Eshelby's ellipsoidal inclusion method, as documented in $[25,26]$, was often used to study the elastic fields in presence of a deformation twin, as done in [27-29] for several materials. Previous studies using this formalism [27-29] or recent full-field Fast Fourier transform-based calculations [30] essentially focus on primary twins. It is noteworthy that the internal stress field due to classic CTW-ETW double twinning was calculated in the framework of a double inclusion micromechanical model in [31]. Here, a double inclusion model (Fig. 6) that allows analytical free energy calculations is developed and applied to sequential ETWETW double twinning as observed experimentally in the present study.

\subsection{Double inclusion based-micromechanical modeling of double twinning}

Analytical free energy calculations are performed through a double inclusion model representing a double twin with twinning shears in the primary and the secondary twins considered as "eigenstrains", homogeneous elasticity and overall plasticity around the double twin. The primary twin will be considered as an ellipsoidal inclusion that contains a secondary twin of ellipsoidal shape.

Let us consider two ellipsoids $V_{A}$ and $V_{B}$ embedded in an infinite matrix $V$, with $V_{B} \subseteq V_{A} \subset V$ as shown in Fig. 6. The elastic properties of $V_{A}, V_{B}$ and $V$ are described by a homogeneous elastic tensor C. For double twinning, $V_{A}$ and $V_{B}$ refer to the primary and the secondary twins. $\mathbf{E}^{\mathbf{p}}, \boldsymbol{\varepsilon}_{\mathbf{a}}^{\mathbf{p}}$ and $\boldsymbol{\varepsilon}_{\mathbf{b}}^{\mathbf{p}}$ are uniform plastic strains in sub-domains $V-V_{A}, V_{A}-V_{B}$ and $V_{B}$, respectively. $\mathbf{E}^{\mathbf{p}}$ is the 


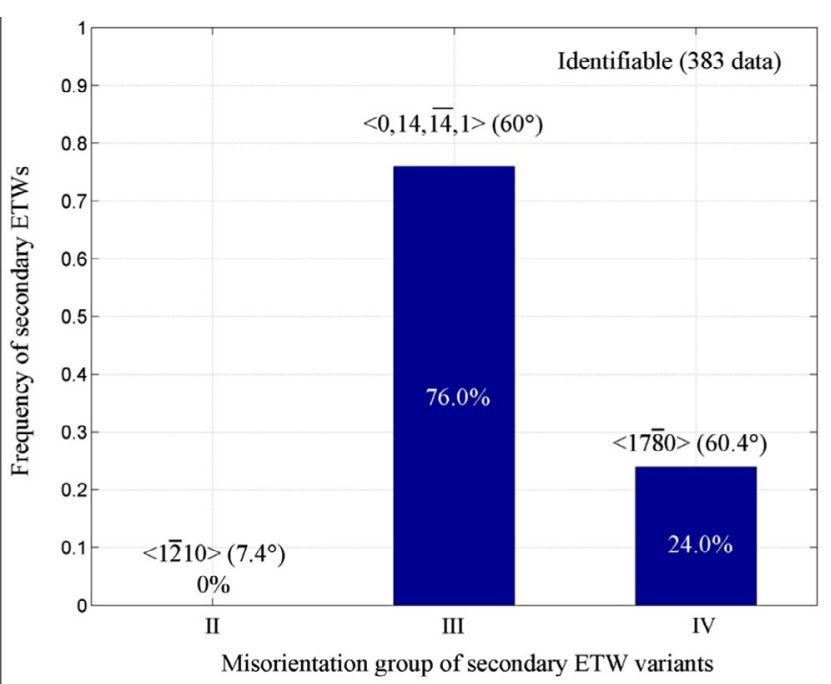

(a)

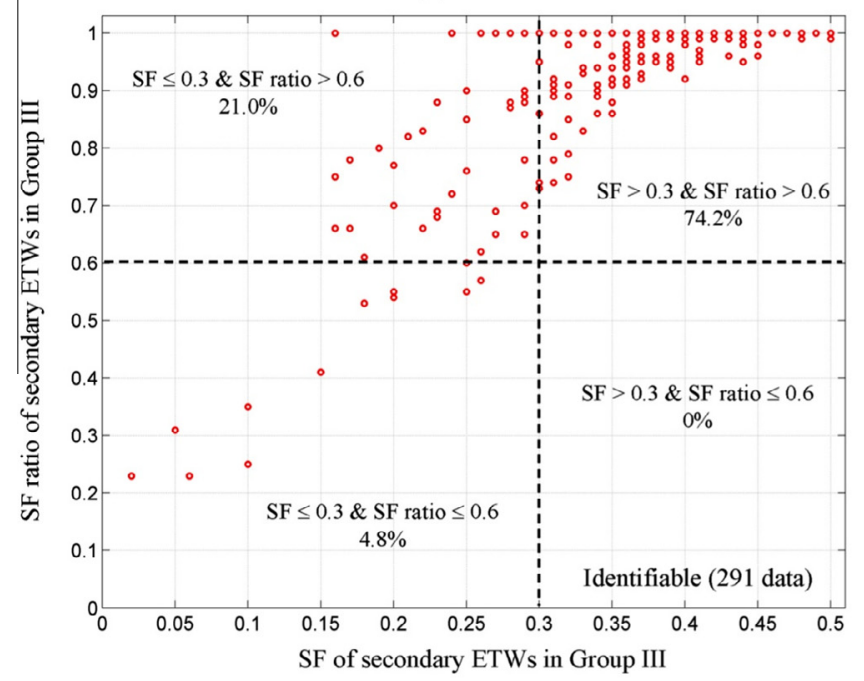

(b)

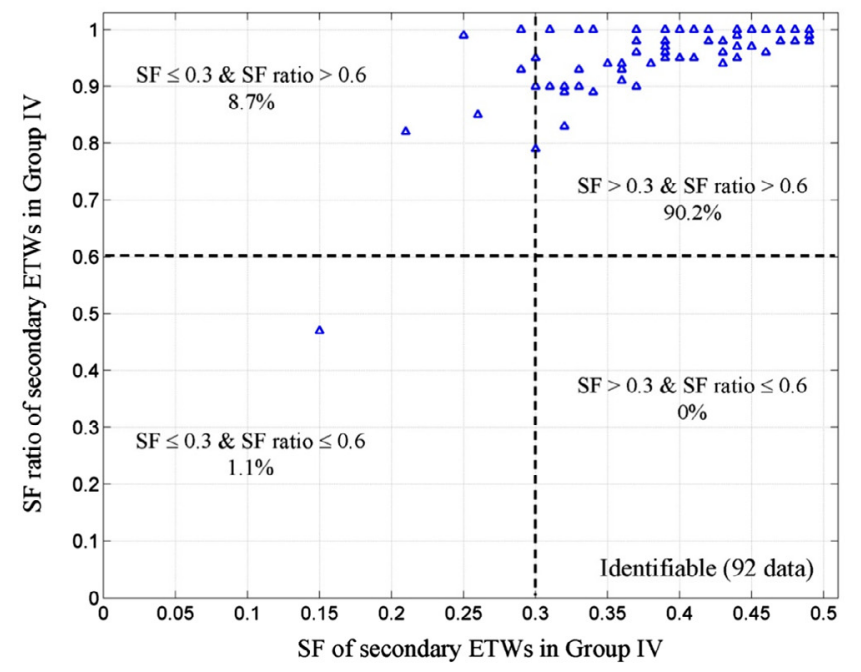

(c)

Fig. 5. For 383 identifiable secondary ETWs: (a) Frequency of the twins in terms of their misorientation groups, (b) SFs and SF ratios of 291 twins in Group III, (c) SFs and SF ratios of 92 twins in Group IV. macroscopic plastic strain tensor undergone by the specimen. Let us now consider that primary twinning occurred first in $V_{A}$, followed by secondary twinning in $V_{B}$ and, for the sake of simplicity, that no other plastic deformation mechanism due to crystallographic slip took place in $V_{A} . \varepsilon_{1}^{\mathbf{p}}$ and $\boldsymbol{\varepsilon}_{2}^{\mathbf{p}}$ denote the respective plastic strain tensors induced by primary and secondary twinning shears. Therefore (Fig. 6),

$\boldsymbol{\varepsilon}_{\mathbf{a}}^{\mathbf{P}}=\boldsymbol{\varepsilon}_{1}^{\mathbf{P}}, \quad \boldsymbol{\varepsilon}_{\mathbf{b}}^{\mathbf{P}}=\boldsymbol{\varepsilon}_{1}^{\mathbf{P}}+\boldsymbol{\varepsilon}_{2}^{\mathbf{P}}$.

The Helmholtz free energy per unit volume for the whole system $V$ usually depends on the volume density of elastic energy and other contributions like stacking fault energy, interfacial energy, etc. In this study, only the elastic energy per unit volume is considered and defined as (see e.g. [32]):

$\Phi^{\mathrm{II}}=\frac{1}{2 V} \int_{V} \boldsymbol{\sigma}(r): \boldsymbol{\varepsilon}^{\mathbf{e}}(r) \mathrm{d} r$,

where the superscript "II" indicates that energy is computed after secondary twinning (i.e., the final configuration), the operator ":" is the contracted product between two tensors, $\boldsymbol{\sigma}(r)$ and $\boldsymbol{\varepsilon}^{\mathbf{e}}(r)$ are respectively the Cauchy stress and the elastic strain tensors at any given position vector " $r$ ". Using stress equilibrium (without body forces) and the divergence theorem, this energy can be expressed as [32]:

$\Phi^{\mathrm{II}}=\frac{1}{2} \boldsymbol{\Sigma}: \mathbf{E}-\frac{1}{2 V} \int_{V} \boldsymbol{\sigma}(r): \boldsymbol{\varepsilon}^{\mathbf{p}}(r) \mathrm{d} r$,

where $\mathbf{E}$ is the imposed homogeneous strain tensor on the boundary $\partial V$ of $V$ and $\boldsymbol{\Sigma}=\frac{1}{V} \int_{V} \boldsymbol{\sigma}(r) \mathrm{d} r=\mathbf{C}:\left(\mathbf{E}-\mathbf{E}^{\mathbf{p}}\right)$ is the macroscopic stress tensor of the system, i.e., the average stress tensor over $V$. Then, following [33], $\Phi^{\mathrm{II}}$ can be approximated by using the average stress tensors, $\overline{\boldsymbol{\sigma}}^{V-V_{A}}, \overline{\boldsymbol{\sigma}}^{V_{A}-V_{B}}$ and $\overline{\boldsymbol{\sigma}}^{V_{B}}$ in sub-domains $V-V_{A}, V_{A}-V_{B}$ and $V_{B}$, respectively:

$$
\Phi^{\mathrm{II}}=\frac{1}{2} \boldsymbol{\Sigma}: \mathbf{E}-\frac{1}{2 V}\left[\left(V-V_{A}\right) \overline{\boldsymbol{\sigma}}^{V-V_{A}}: \mathbf{E}^{\mathbf{p}}+\left(V_{A}-V_{B}\right) \overline{\boldsymbol{\sigma}}^{V_{A}-V_{B}}: \boldsymbol{\varepsilon}_{\mathbf{a}}^{\mathbf{p}}+V_{B} \overline{\boldsymbol{\sigma}}^{V_{B}}: \boldsymbol{\varepsilon}_{\mathbf{b}}^{\mathbf{p}}\right]
$$

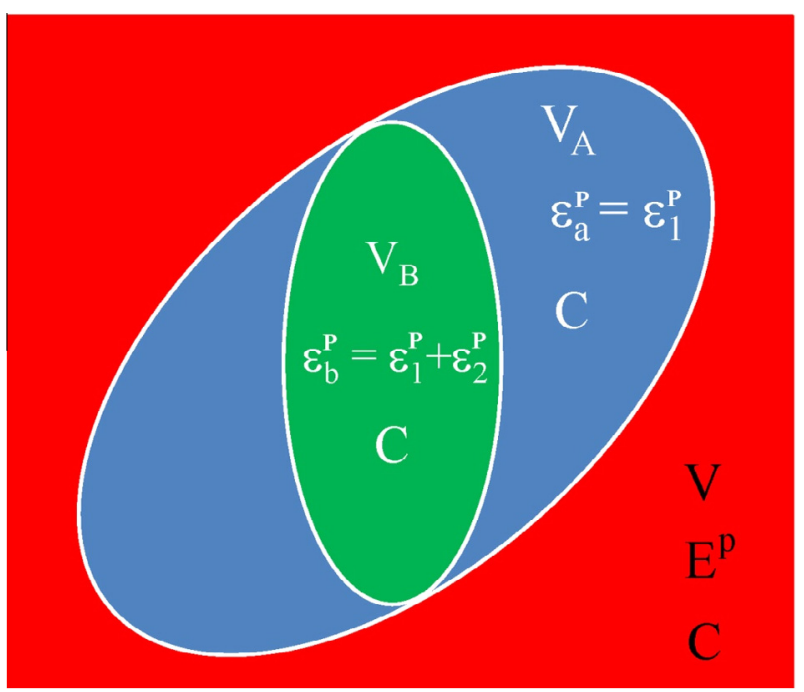

Fig. 6. Schematic representation of double twin inclusion containing two ellipsoidal inclusions $V_{A}$ and $V_{B}$ in an infinite matrix $V\left(V_{B} \subseteq V_{A} \subset V\right)$ with a homogeneous tensor $\mathbf{C}$ of elastic moduli. Plastic strains ("eigenstrains") are denoted as $\mathbf{E}^{\mathbf{p}}$, $\varepsilon_{\mathrm{a}}^{\mathbf{p}}$ and $\varepsilon_{\mathrm{b}}^{\mathbf{p}}$, which are prescribed within $V-V_{A}, V_{A}-V_{B}$ and $V_{B}$, respectively. $\varepsilon_{1}^{\mathbf{p}}$ and $\varepsilon_{2}^{\mathbf{p}}$ denote the respective twinning "eigenstrain" (or stress-free strain) tensors due to twinning shear induced by primary and secondary twinning in a given frame that will be specified later. 
The average stress tensor over $V_{A}-V_{B}$ is derived using the Tanaka-Mori scheme [26,33] as detailed in the Appendix A. Their substitution in Eq. (4) and some algebraic manipulations lead to the following expression of $\Phi^{\mathrm{II}}$ :

$$
\begin{aligned}
& \Phi^{\mathrm{II}}=\frac{1}{2} \boldsymbol{\Sigma}:\left(\mathbf{E}-\mathbf{E}^{\mathbf{p}}\right)-\frac{1}{2} \frac{V_{A}}{V}\left\{\boldsymbol{\Sigma}:\left(\boldsymbol{\varepsilon}_{1}^{\mathbf{P}}-\mathbf{E}^{\mathbf{p}}\right)+\mathbf{C}:\left(\mathbf{S}^{V_{A}}-\mathbf{I}\right):\left(\boldsymbol{\varepsilon}_{1}^{\mathbf{P}}-\mathbf{E}^{\mathbf{p}}\right): \boldsymbol{\varepsilon}_{1}^{\mathbf{P}}\right\} \\
& -\frac{1}{2} \frac{V_{B}}{V}\left\{\boldsymbol{\Sigma}: \boldsymbol{\varepsilon}_{2}^{\mathbf{P}}+\mathbf{C}:\left[\left(\mathbf{S}^{V_{A}}-\mathbf{I}\right):\left(\boldsymbol{\varepsilon}_{1}^{\mathbf{P}}: \boldsymbol{\varepsilon}_{2}^{\mathbf{P}}+\boldsymbol{\varepsilon}_{2}^{\mathbf{P}}: \boldsymbol{\varepsilon}_{1}^{\mathbf{P}}-\mathbf{E}^{\mathbf{P}}: \boldsymbol{\varepsilon}_{2}^{\mathbf{P}}\right)+\left(\mathbf{S}^{V_{B}}-\mathbf{I}\right): \boldsymbol{\varepsilon}_{2}^{\mathbf{P}}: \boldsymbol{\varepsilon}_{2}^{\mathbf{P}}\right]\right\},
\end{aligned}
$$

where $\boldsymbol{S}^{V_{A}}$ and $\boldsymbol{S}^{V_{B}}$ are uniform Eshelby tensors $[25,26]$ for interior points in ellipsoidal inclusions $V_{A}$ and $V_{B}$, respectively. The elastic energy $\Phi^{\mathrm{I}}$ after primary twinning and before secondary twinning occurs inside $V_{A}$ is obtained by taking $\varepsilon_{2}^{\mathbf{p}}=0$ or $V_{B}=0$ in Eq. (5). Therefore, the change of elastic energy density due to secondary twinning reads:

$$
\begin{aligned}
\Delta \Phi= & \Phi^{\mathrm{II}}-\Phi^{\mathrm{I}} \\
= & -\frac{1}{2} \frac{V_{B}}{V}\left\{\boldsymbol{\Sigma}: \boldsymbol{\varepsilon}_{2}^{\mathbf{P}}+C:\left[\left(\mathbf{S}^{V_{A}}-I\right):\left(\boldsymbol{\varepsilon}_{1}^{\mathbf{P}}: \boldsymbol{\varepsilon}_{2}^{\mathbf{P}}+\boldsymbol{\varepsilon}_{2}^{\mathbf{P}}: \boldsymbol{\varepsilon}_{1}^{\mathbf{P}}-\mathbf{E}^{\mathbf{p}}: \boldsymbol{\varepsilon}_{2}^{\mathbf{P}}\right)\right.\right. \\
& \left.\left.+\left(\mathbf{S}^{V_{B}}-\mathbf{I}\right): \boldsymbol{\varepsilon}_{2}^{\mathbf{P}}: \boldsymbol{\varepsilon}_{2}^{\mathbf{P}}\right]\right\} .
\end{aligned}
$$

The expression of $\Delta \Phi$ includes relative shape effect of the primary and the secondary twins through the Eshelby tensors $\mathbf{S}^{V_{A}}$ and $\mathbf{S}^{V_{B}}$, which are different from each other when $V_{A}$ and $V_{B}$ are non homothetic.

\subsection{Application to ETW-ETW double twinning}

In this section, the micromechanical double inclusion model is applied to the sequential ETW-ETW double twinning in Mg. The general configuration will be specified, followed by a representative example.

\subsubsection{General configuration}

The assumption of homogeneous isotropic elasticity is a good approximation for $\mathrm{Mg}$ due to its very low elastic anisotropy [34]. Here, the elastic shear moduli and Poisson ratio used for the calculations are respectively: $\mu=17 \mathrm{GPa}$ and $v=0.29$. As shown in Fig. 7a, inclusions $V_{A}$ and $V_{B}$ representing twins are assumed to have oblate shapes, i.e., the main axes $a_{1}=a_{2}>a_{3}$ for $V_{A}$ and $b_{1}=b_{2}>b_{3}$ for $V_{B}$. The twinning reference frame of $V_{A}$ or $V_{B}$ is defined by $x_{\mathrm{tw}} / /$ shear direction (SD), $y_{\mathrm{tw}} / /$ shear plane normal (SPN), and $z_{\mathrm{tw}} / /$ twinning plane normal (TPN), as in Fig. 7a. The main axis $a_{3}$ (or $b_{3}$ ) is parallel to $z_{\mathrm{tw}}$ of $V_{A}$ (or $V_{B}$ ), while another main axis $a_{1}$ (or $b_{1}$ ) is parallel to $x_{\mathrm{tw}}$ of $V_{A}$ (or $V_{B}$ ). Since the main axes $a_{1}=a_{2}$, the planar section normal to $a_{3}$ has a circular shape with radius $a_{1}$, which is analogous for $V_{B}$. For clarity, Fig. 7a is a projection along $y_{\mathrm{tw}}$ of $V_{A}$ and the corresponding projection of $V_{B}$ is represented by dashed lines. In Fig. 7a, there is a swelling effect of $V_{B}$ due to the projection unless $y_{\mathrm{tw}}$ of $V_{B}$ is parallel to $y_{\mathrm{tw}}$ of $V_{A}$.

The interior Eshelby tensor $\mathbf{S}^{V_{A}}$ (or $\boldsymbol{S}^{V_{B}}$ ) is related to the aspect ratio of $V_{A}$ (or $V_{B}$ ). Assuming that the main axis $b_{1}$ of $V_{B}$ is constrained by $V_{A}$, it can be deduced from geometry (Fig. 7a) that

$b_{1}=a_{1} /\left[\cos ^{2} \theta+\left(\sin \theta / r_{A}\right)^{2}\right]^{1 / 2}$,

where the aspect ratio $r_{A}=a_{3} / a_{1}$ and $\theta$ is the angle between the twinning planes of $V_{A}$ and $V_{B}$. Then, it can be deduced that the aspect ratio of $V_{B}$ is

$r_{B}=b_{3} / b_{1}=f_{V B} r_{A}\left[\cos ^{2} \theta+\left(\sin \theta / r_{A}\right)^{2}\right]^{3 / 2}$,

where $f_{V B}=V_{B} / V_{A}$ is the volume fraction of $V_{B}$. With given $f_{V B}$ and $r_{A}$, the smaller the $\theta$ angle, the smaller the $r_{B}$. The values of $\theta$ angles for double ETW variants in Groups I-IV are $0^{\circ}, 86.3^{\circ}, 40.0^{\circ}$ and $72.6^{\circ}$, respectively (Fig. 3b). Therefore, the corresponding $r_{B}$ values can be ordered increasingly as follows: Groups I $<$ Groups III $<$ Groups IV $<$ Groups II.

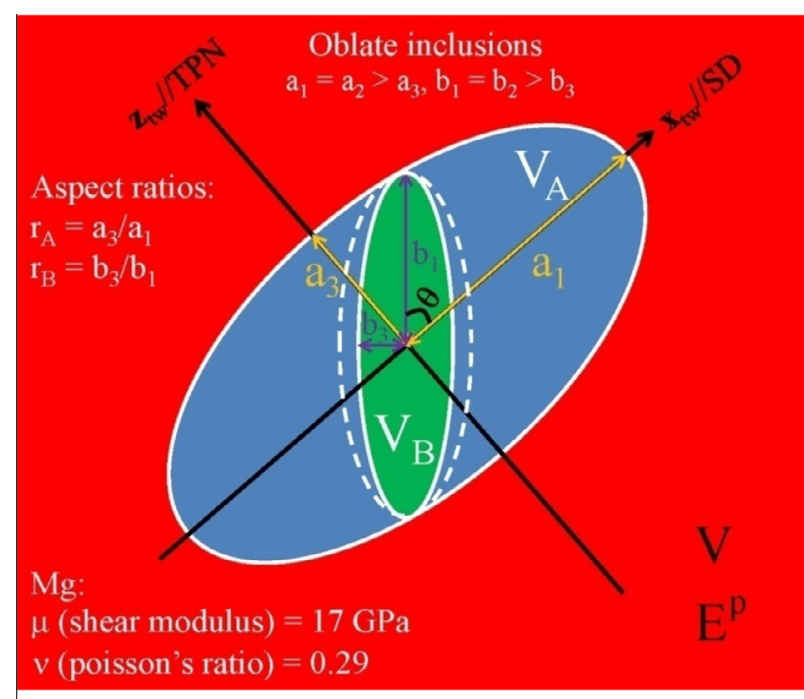

(a)

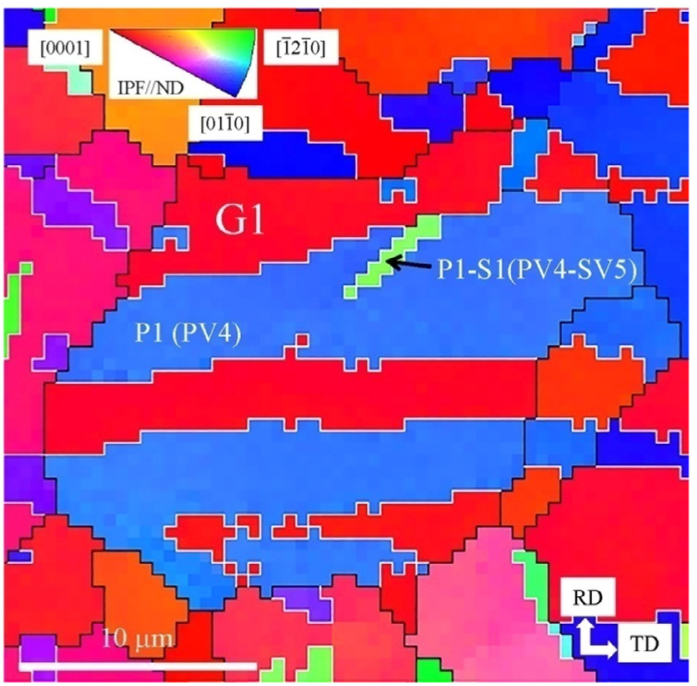

(b)

Fig. 7. (a) Configuration of the double inclusion modeling for ETW-ETW double twinning. Twins $V_{A}$ and $V_{B}$ have oblate spheroidal shapes $\left(a_{1}=a_{2}>a_{3}\right.$ and $\left.b_{1}=b_{2}>b_{3}\right)$. The twinning reference frame of $V_{A}$ or $V_{B}$ is defined by $x_{\mathrm{tw}} / /$ shear direction (SD), $y_{\mathrm{tw}} / /$ shear plane normal (SPN), and $z_{\mathrm{tw}} / /$ twinning plane normal (TPN). This figure is a projection along $y_{\mathrm{tw}}$ of $V_{A}$. The dashed line is the trace of $V_{B}$ after projection. The angle between the twinning planes of $V_{A}$ and $V_{B}$ is $\theta$. (b) A representative EBSD microstructure showing sequential ETW-ETW double twinning, serving as an example of the modeling.

Secondary twinning is supposed to happen at the beginning of the second TD compression. $\mathbf{E}^{\mathbf{p}}$ is thus considered as the plastic strain tensor associated with the first RD compression, whose diagonal components are determined experimentally to be $E_{R D}^{P} \approx-0.018, E_{T D}^{P} \approx 0$, and $E_{N D}^{P} \approx 0.018$. The shear magnitude of an ETW in Mg is 0.129, which can be used to compute both tensors $\boldsymbol{\varepsilon}_{1}^{\mathbf{p}}$ and $\boldsymbol{\varepsilon}_{2}^{\mathbf{p}}$. Using Eq. (6), we can investigate the change of free energy density due to secondary twinning in order to determine the selection of secondary ETW variant. Therefore, the selected variants are considered as the ones minimizing the energy change (lowest free energy criterion). A typical example (Fig. 7b) will be given, from which general conclusions will be addressed.

\subsubsection{A representative example}

A representative EBSD microstructure showing sequential ETW-ETW double twinning is presented in Fig. 7b. This figure 
shows a secondary twin G1-P1-S1 in its parent primary twin G1-P1 in the grain G1. The variant of the secondary twin G1-P1-S1 is PV4-SV5 (Table 2). Then, G1, G1-P1 and G1-P1-S1 correspond to $V, V_{A}$ and $V_{B}$ in Fig. $7 \mathrm{a}$, respectively. The Euler angles of G1 are $\left(112.43^{\circ}, 168.2^{\circ}, 33.11^{\circ}\right)$. An estimate of the aspect ratio of the primary twin G1-P1 is $r_{A}=0.2$ from Fig. $7 \mathrm{~b}$. According to the same figure, the surface fraction of G1-P1-S1 with respect to G1-P1 is about $f_{\text {surface }}=0.03$. Using the assumption of oblate twin shape (Fig. 7a), it can be deduced that $f_{V B}=\left[b_{1} b_{3} /\left(a_{1} a_{3}\right)\right]\left(b_{1} / a_{1}\right)$. The term $b_{1} b_{3} /\left(a_{1} a_{3}\right)$ is approximately equal to $f_{\text {surface, }}$ and then it can be roughly estimated that $f_{V B}=0.01$. Since the value of $\theta$ is known for any possible secondary ETW variant (Fig. 3b), Eq. (8) provides the ratio $r_{B}$ of any possible secondary ETW variant according to the present double twinning assumption and for the same estimates of $f_{V B}$ and $r_{A}$.

Fig. 8a exhibits the normalized free energy density change $\Delta d$ $\left(=\Delta \phi V / V_{B}\right)$ for a unit volume $V=1$ due to secondary twinning as a function of the applied TD stress $\Sigma_{\mathrm{TD}}$, which increases from $0 \mathrm{MPa}$ to the yield strength of $120 \mathrm{MPa}$ (Fig. 1a). First of all, when $\Sigma_{\mathrm{TD}}=0 \mathrm{MPa}, \Delta d$ values are ranked from the lowest to the highest as follows: PV4-SV4 (Group I, detwinning) < PV4-SV1 (Group II), PV4-SV3 and PV4-SV5 (Group III) < PV4-SV2 and PV4-SV6 (Group

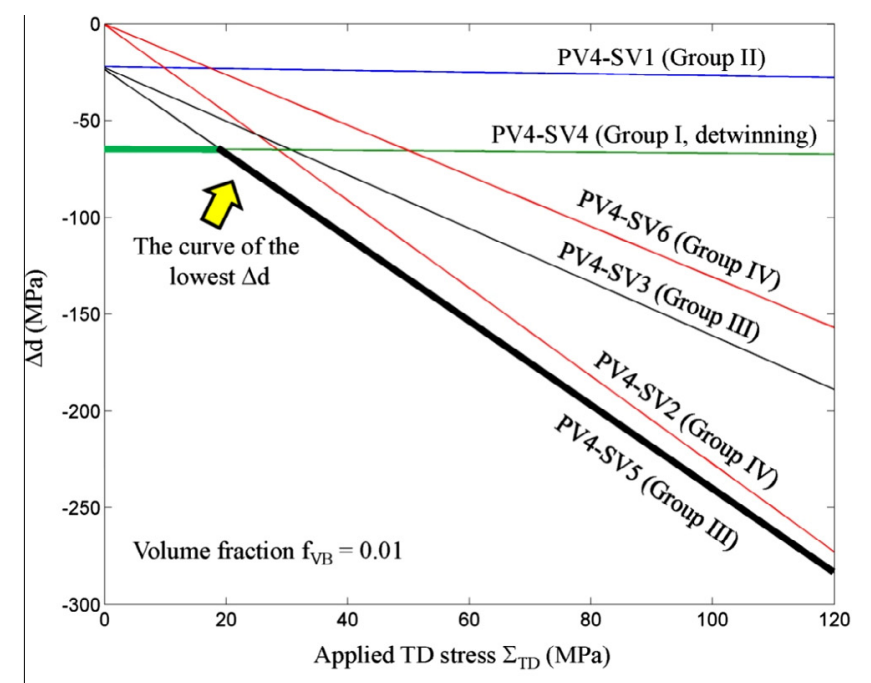

(a)

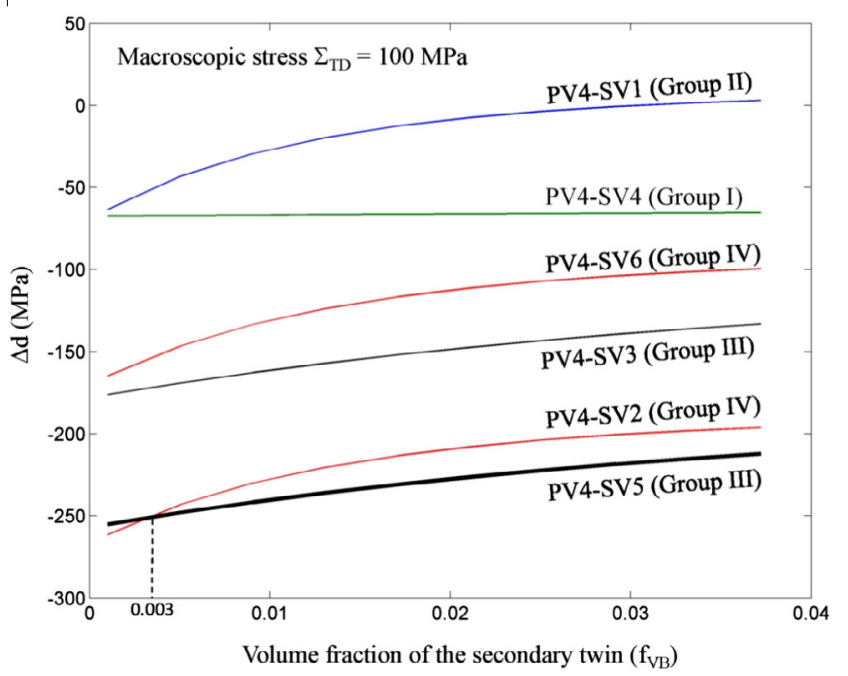

(b)

Fig. 8. The normalized free energy density change $\Delta d\left(=\Delta \phi V / V_{B}\right.$, with $\left.V=1\right)$ due to secondary twinning: (a) as a function of the applied TD stress $\Sigma_{\mathrm{TD}}$; (b) as a function of the volume fraction of the secondary twin $\left(f_{\mathrm{VB}}\right)$.
IV). Second, when $\Sigma_{\mathrm{TD}}$ reaches $\sim 20 \mathrm{MPa}$, a transition from PV4-SV4 (detwinning) to PV4-SV5 (Group III) is expected from Fig. 8a, as highlighted with the arrow reported on this figure indicating the transition and the thick solid line representing the lowest energy curve. Practically, the macroscopic stress is higher than the linear elastic limit, i.e., $\Sigma_{\mathrm{TD}}>\sim 80 \mathrm{MPa}$ (Fig. 1a), when a secondary twin is observed. Fig. 8a shows that PV4-SV5 (Group III) remains as the variant with the lowest $d$ values when $\Sigma_{\mathrm{TD}}>80 \mathrm{MPa}$. This result is consistent with the experimental observation at the end of the second TD compression (Fig. 7b). It is noteworthy that the $d$ values for PV4-SV1 and PV4-SV4 are almost unchanged with increasing the overall stress $\Sigma_{\mathrm{TD}}$ since the term $\boldsymbol{\sigma}: \boldsymbol{\varepsilon}_{2}^{\mathbf{P}} \approx 0$ in Eq. (6) in both cases.

Let us recall that it was highlighted in Section 3.4 that a classical (macroscopic) SF based analysis cannot explain the preference of variants in Group III over those in Group IV. The SFs of the possible secondary twin variants associated with the primary twin G1-P1 in Fig. 7b can be ranked from the highest to the lowest as follows: 0.43 (PV4-SV2, Group IV) $>0.42$ (PV4-SV5, Group III) $>0.27$ (PV4-SV3, Group III) > 0.25 (PV4-SV6, Group IV) > 0.009 (PV4-SV1, Group II) $>0.005$ (PV4-SV4, Group I). Although the SF of the observed PV4-SV5 (Group III) is 0.01 lower than that of PV4-SV2 (Group IV), the $d$ values of the former one are about $23 \mathrm{MPa}$ to $11 \mathrm{MPa}$ lower than those of the latter one with increasing $\Sigma_{\mathrm{TD}}$. This explains the reason why PV4-SV5 (Group III) with a lower SF is preferred over PV4-SV2 (Group IV). When $\Sigma_{\mathrm{TD}}>\sim 18 \mathrm{MPa}$, the $d$ value of PV4-SV1 (Group II) is the highest so that it should be the least preferred. Let us recall that none of the identified double extension twins belongs to Group II (Fig. 5a), which is consistent with the present micromechanical calculations.

Fig. 8b exhibits the evolution of $d$ as a function of the volume fraction of the secondary twin $\left(f_{V B}\right)$. The macroscopic stress is set to be $100 \mathrm{MPa}$ (i.e., $\Sigma_{\mathrm{TD}}>80 \mathrm{MPa}$ ), corresponding to the elasticplastic transition (Fig. 1a). It is noteworthy that the chosen level of the macroscopic stress will not modify the trend given by Fig. $8 \mathrm{~b}$ but only the $d$ magnitudes. Except the $d$ value of PV4-SV4 (Group I, detwinning) which is nearly constant, those of the other variants increase with the volume fraction. When $f_{V B}>0.003$, the $d$ value of the observed PV4-SV5 (Group III) remains the lowest, and thus the most preferred. By contrast, that of PV4-SV1 (Group II) is always the highest and increases the most rapidly with the volume fraction.

\subsection{Discussion}

According to the present model, for reasonable values of applied stress and secondary twin volume fraction, when the SFs of two secondary twin variants belonging separately to Groups III and IV are comparable, the one in Group III always leads to a noticeably larger decrease of free energy density change. This explains why the variants in Group III are predominant in the microstructure (Fig. 5a). In addition, the changes of free energy density of variants in Group II are constantly high and increase the most rapidly during growth, which is in agreement with the fact that none of them was identified experimentally (Fig. 5a).

The developed micromechanical static double-inclusion model considers the following new aspects: (i) the elastic interaction between the primary and the secondary twins; (ii) the relative shape effect of the primary and the secondary twins; (iii) the plastic deformation in the grain matrix due to the first loading. Although the current static approach does not account for plastic accommodation, it underlines the key importance of the double-inclusion topology in order to successfully explain the preference of secondary twins in Group III. Actually, the classical Eshelby's single-inclusion model (i.e., assuming that the secondary 
twin is embedded within the infinite primary twin matrix) has also been tried. However, it fails to reproduce this preference.

Besides, according to Fig. $8 \mathrm{~b}$, detwinning seems to be the predominant mechanism among all the secondary twinning possibilities at low stresses when $\Sigma_{\mathrm{TD}}<\sim 20 \mathrm{MPa}$. This result does not necessarily imply that detwinning will occur at such low stresses because the CRSS associated with ETW has still to be overcome. However, this micromechanical result is interesting since detwinning has been frequently observed during unloading (i.e., low stress levels) [35-39].

\section{Conclusions}

In previous works on twinning in Mg alloys, $\{10-12\}-\{10-12\}$ double extension twins have been much less studied. In this work, a two-step plastic deformation (i.e., 1.8\% compression along $\mathrm{RD}+1.3 \%$ compression along TD) has been applied on AZ31 cubes in order to generate sequential double extension twins. Combined statistical and micromechanical studies have been conducted to explain their variant selection. The EBSD measurements cover a large area of $0.82 \mathrm{~mm}^{2}$, containing 4481 grains, 11,052 primary ETWs, and 585 secondary ETWs. The secondary ETWs taking up an area fraction of $0.42 \%$ form in the second TD compression, while the primary ETWs taking up an area fraction of $27.94 \%$ form in both the RD and the TD compressions. The variant types of $383 \mathrm{sec}-$ ondary ETWs are unambiguously identified. The study of these identifiable secondary ETWs leads to the following conclusions:

1. All of the secondary ETWs have positive SFs. 78.1\% of them have SFs $>0.3$, and $95.8 \%$ of them have SF ratios $>0.6$. The majority of the secondary ETWs are the variants with relatively high SFs compared with the other possible variants in their parent primary ETWs.

2. The 36 possible double ETW variants can be classified into Groups I to IV according to their misorientations with respect to the grain matrix. Twins in Group I (corresponding to detwinning) are not observable in this study. For the other groups, twins in Group III are predominant (i.e., 76.0\%), while no twin in Group II is found. The observed twins in Groups III and IV have relative high SFs. However, the SF criterion cannot explain why twins in Group III are preferred over their nearest orientated neighbors in Group IV, since their SF differences are negligible.

3. A micromechanics-based double inclusion model has been developed to analytically compute the elastic free energy change due to the occurrence of secondary twin in a final sequential double twinning configuration. It is revealed that the free energy density change of a twin in Group III exhibits the lowest value, especially compared to the case of a twin in Group IV. Thus, an energy-based criterion based on a double-inclusion topology make it possible to explain why twins in Group III are predominant, which was impossible with a classic SF analysis.

\section{Acknowledgements}

The authors thank Dr. Edgar F. Rauch (SIMAP, University Grenoble/CNRS) for fruitful discussions regarding twinning mechanisms in Mg alloys. The support of the French National Research Agency under the project MAGTWIN (referenced as ANR-12-BS09-0010-02) is gratefully acknowledged. This work is also supported by the French State through the National Research Agency (ANR) under the program Investment in the future (LabEx DAMAS referenced as ANR-11-LABX-0008-01).

\section{Appendix A}

The general expression of total strain for a given distribution of plastic strain $\boldsymbol{\varepsilon}^{\mathbf{P}}(\boldsymbol{r})$ is given in homogenous elasticity by [40]

$\boldsymbol{\varepsilon}(r)=\mathbf{E}+\int_{V} \Gamma^{\mathbf{s}}\left(r-r^{\prime}\right): \mathbf{C}: \boldsymbol{\varepsilon}^{\mathbf{P}}\left(r^{\prime}\right) \mathrm{d} r^{\prime}$,

where $\Gamma^{\mathbf{s}}$ is the symmetric modified Green tensor defined as $\Gamma_{\mathrm{ijkl}}^{\mathrm{s}}\left(r-r^{\prime}\right)=-\frac{1}{2}\left(\mathrm{G}_{\mathrm{ik}, \mathrm{jl}}\left(r-r^{\prime}\right)+\mathrm{G}_{\mathrm{jk}, \mathrm{il}}\left(r-r^{\prime}\right)\right)$ and $\mathbf{G}$ is the elastic Green function [26]. According to Fig. 6, the plastic strain of any position vector " $r$ " in $V$ can be described as:

$\boldsymbol{\varepsilon}^{\mathbf{P}}(\mathbf{r})=\left(\boldsymbol{\varepsilon}_{\mathbf{b}}^{\mathbf{P}}-\boldsymbol{\varepsilon}_{\mathbf{a}}^{\mathbf{P}}\right) \theta_{B}(r)+\boldsymbol{\varepsilon}_{\mathbf{a}}^{\mathbf{P}} \theta_{A}(r)+\left(1-\theta_{A}(r)\right) \mathbf{E}^{\mathbf{p}}$,

where $\theta_{B}(\mathrm{r})$ and $\theta_{A}(\mathrm{r})$ are characteristic functions associated with $V_{B}$ and $V_{A}$, respectively. These functions are equal to 1 inside and 0 outside their corresponding volumes, respectively. Then, Eq. (A1) becomes:

$$
\begin{aligned}
\boldsymbol{\varepsilon}(\mathbf{r})= & \mathbf{E}+\int_{V_{B}} \boldsymbol{\Gamma}^{\mathbf{s}}\left(r-r^{\prime}\right): \mathbf{C}:\left(\boldsymbol{\varepsilon}_{\mathbf{b}}^{\mathbf{p}}-\boldsymbol{\varepsilon}_{\mathbf{a}}^{\mathbf{p}}\right) \mathrm{d} r^{\prime}+\int_{V_{A}} \Gamma^{\mathbf{s}}\left(r-r^{\prime}\right): \mathbf{C}: \boldsymbol{\varepsilon}_{\mathbf{a}}^{\mathbf{p}} \mathrm{d} r^{\prime} \\
& -\int_{V_{A}} \Gamma^{\mathbf{s}}\left(r-r^{\prime}\right): \mathbf{C}: \mathbf{E}^{\mathbf{p}} \mathrm{d} r^{\prime} .
\end{aligned}
$$

For ellipsoidal inclusions $V_{A}$ and $V_{B}$, their interior Eshelby tensors $\mathbf{S}^{V_{A}}$ and $\mathbf{S}^{V_{B}}$ are uniform, which are defined as [25,26]:

$\mathbf{S}^{V_{A}}=\int_{V_{A}} \boldsymbol{\Gamma}^{\mathbf{s}}\left(r-r^{\prime}\right): \mathbf{C d} \mathbf{r}^{\prime}, \quad \mathbf{S}^{V_{B}}=\int_{V_{B}} \boldsymbol{\Gamma}^{\mathbf{s}}\left(r-r^{\prime}\right): \mathbf{C d} r^{\prime}$.

From Eqs. (A3) and (A4), the total strain in $V_{B}$ is uniform and is given by

$\boldsymbol{\varepsilon}\left(r \in V_{B}\right)=\mathbf{E}+\mathbf{S}^{V_{B}}:\left(\boldsymbol{\varepsilon}_{\mathbf{b}}^{\mathbf{p}}-\boldsymbol{\varepsilon}_{\mathbf{a}}^{\mathbf{p}}\right)+\mathbf{S}^{V_{A}}:\left(\boldsymbol{\varepsilon}_{\mathbf{a}}^{\mathbf{p}}-\mathbf{E}^{\mathbf{p}}\right)=\overline{\boldsymbol{\varepsilon}}^{V_{B}}$.

The volume strain average over $V_{A}-V_{B}$ results from the application of the Tanaka-Mori's result [9]:

$\overline{\boldsymbol{\varepsilon}}^{V_{A}-V_{B}}=\mathbf{E}+\mathbf{S}^{V_{A}}:\left(\boldsymbol{\varepsilon}_{\mathbf{a}}^{\mathbf{p}}-\mathbf{E}^{\mathbf{p}}\right)-\frac{V_{B}}{V_{A}-V_{B}}\left(\mathbf{S}^{V_{B}}-\mathbf{S}^{V_{A}}\right):\left(\boldsymbol{\varepsilon}_{\mathbf{b}}^{\mathbf{p}}-\boldsymbol{\varepsilon}_{\mathbf{a}}^{\mathbf{p}}\right)$.

Finally, the average stresses in sub-domains $V-V_{A}, V_{A}-V_{B}$ and $V_{B}$, are directly deduced from $\overline{\boldsymbol{\sigma}}^{V_{A}-V_{B}}=\mathbf{C}:\left(\overline{\boldsymbol{\varepsilon}}^{V_{A}-V_{B}}-\boldsymbol{\varepsilon}_{\mathbf{a}}^{\mathbf{p}}\right)$, $\overline{\boldsymbol{\sigma}}^{V_{B}}=\mathbf{C}:\left(\overline{\boldsymbol{\varepsilon}}^{V_{B}}-\boldsymbol{\varepsilon}_{\mathbf{b}}^{\mathbf{p}}\right)$ and $\overline{\boldsymbol{\sigma}}^{V-V_{A}}=\boldsymbol{\Sigma}$, since $V$ is an infinite medium and the fraction of $V_{A}$ inside $V$ is negligible such that $\boldsymbol{\Sigma}=\frac{1}{V} \int_{V} \boldsymbol{\sigma}(r) \mathrm{d} r=\mathbf{C}:\left(\mathbf{E}-\mathbf{E}^{\mathbf{p}}\right)$ is the remote stress at the boundary of $V$.

\section{References}

[1] J.W. Christian, S. Mahajan, Deformation twinning, Prog. Mater. Sci. 39 (1995) $1-157$.

[2] M.D. Nave, M.R. Barnett, Microstructures and textures of pure magnesium deformed in plane-strain compression, Scr. Mater. 51 (2004) 881-885.

[3] M.R. Barnett, Twinning and the ductility of magnesium alloys Part I: "Tension" twins, Mater. Sci. Eng. A 464 (2007) 1-7.

[4] S.G. Hong, S.H. Park, C.S. Lee, Role of 10-12 twinning characteristics in the deformation behavior of a polycrystalline magnesium alloy, Acta. Mater. 58 (2010) 5873-5885.

[5] S.H. Park, S.G. Hong, C.S. Lee, Activation mode dependent 10-12 twinning characteristics in a polycrystalline magnesium alloy, Scr. Mater. 62 (2010) 202-205.

[6] M.R. Barnett, Z. Keshavarz, A.G. Beer, X. Ma, Non-Schmid behaviour during secondary twinning in a polycrystalline magnesium alloy, Acta. Mater. 56 (2008) 5-15.

[7] E. Martin, L. Capolungo, L. Jiang, J.J. Jonas, Variant selection during secondary twinning in Mg-3\%Al, Acta. Mater. 58 (2010) 3970-3983.

[8] D. Ando, J. Koike, Y. Sutou, Relationship between deformation twinning and surface step formation in AZ31 magnesium alloys, Acta. Mater. 58 (2010) 4316-4324.

[9] M.R. Barnett, Twinning and the ductility of magnesium alloys Part II. "Contraction" twins, Mater. Sci. Eng. A 464 (2007) 8-16.

[10] J. Jain, J. Zou, C.W. Sinclair, J. Poole, Double tensile twinning in a Mg-8Al-0.5Zn alloy, J. Microsc. 242 (2010) 26-36. 
[11] P.G. Partridge, The crystallography and deformation modes of hexagonal closepacked metals, Metall. Rev. Inst. Metals 118 (1967) 169-194.

[12] A. Jager, A. Ostapovets, P. Molnar, P. Lejcek, $\{10-12\}-\{10-12\}$ double twinning in magnesium, Philos. Mag. Lett. 91 (2011) 537-544.

[13] S.H. Park, S.G. Hong, J.H. Lee, C.S. Lee, Multiple twinning modes in rolled Mg3Al-1Zn alloy and their selection mechanism, Mater. Sci. Eng. A 532 (2012) 401-406.

[14] S.H. Park, S.G. Hong, C.S. Lee, In-plane anisotropic deformation behavior of rolled Mg-3Al-1Zn alloy by initial 10-12 twins, Mater. Sci. Eng. A 570 (2013) $149-163$.

[15] Z.X. Long, T.M. Liu, Y.G. Wu, Y. Zhang, Improving the anisotropy of rolled Mg3Al-1Zn alloy by pre-strain and annealing, Mater. Sci. Eng. A 616 (2014) 240245.

[16] F. Bachmann, R. Hielscher, H. Schaeben, Texture analysis with MTEX - free and open source software toolbox, Solid State Phenom. 160 (2010) 63-68.

[17] Oxford Instruments HKL, Oxford channel 5 user manual, Denmark, 2007.

[18] Z.-Z. Shi, Y.D. Zhang, F. Wagner, P.-A. Juan, S. Berbenni, L. Capolungo, J.-S. Lecomte, T. Richeton, On the selection of extension twins with low Schmid factors in a deformed Mg alloy, Acta. Mater. 83 (2015) 17-28.

[19] S.R. Agnew, Ö. Duygulu, Plastic anisotropy and the role of non-basal slip in magnesium alloy AZ31B, Int. J. Plast. 21 (2005) 1161-1193.

[20] S. Xu, T.M. Liu, H.C. Chen, Z.C. Miao, Z. Zhang, W. Zeng, Reducing the tensioncompression yield asymmetry in a hot-rolled $\mathrm{Mg}-3 \mathrm{Al}-1 \mathrm{Zn}$ alloy via multidirectional pre-compression, Mater. Sci. Eng. A 565 (2013) 96-101.

[21] Y.C. Xin, M.Y. Wang, Z. Zeng, M.G. Nie, Q. Liu, Strengthening and toughening of magnesium alloy by 10-12 extension twins, Scr. Mater. 66 (2012) 25-28.

[22] O. Muránsky, D.G. Carr, P. Šittner, E.C. Oliver, In situ neutron diffraction investigation of deformation twinning and pseudoelastic-like behaviour of extruded AZ31 magnesium alloy, Int. J. Plast. 25 (2009) 1107-1127.

[23] B. Clausen, C.N. Tomé, D.W. Brown, S.R. Agnew, Reorientation and stress relaxation due to twinning: modeling and experimental characterization for Mg, Acta. Mater. 56 (2008) 2456-2468.

[24] P.-A. Juan, S. Berbenni, M.R. Barnett, C.N. Tomé, L. Capolungo, A double inclusion homogenization scheme for polycrystals with hierarchal topologies: application to twinning in Mg alloys, Int. J. Plast. 60 (2014) 182-196.

[25] J. Eshelby, The determination of the elastic field of an ellipsoidal inclusion, and related problems, Proc. R Soc. London A 24 (1957) 376-396.

[26] T. Mura, Micromechanics of Defects in Solids, Martinus Nijhoff, Dordrecht, 1987.
[27] R.A. Lebensohn, C.N. Tomé, A study of the stress state associated with twin nucleation and propagation in anisotropic materials, Philos. Mag. A 67 (1993) $187-206$.

[28] M. Kumosa, Strain-energy of a mechanical twin in alpha-iron, J. Phys. D Appl. Phys. 24 (1991) 1816-1821.

[29] J.K. Lee, M.H. Yoo, Elastic strain-energy of deformation twinning in tetragonal crystals, Metall. Trans. A 21 (1990) 2521-2530.

[30] M.A. Kumar, A.K. Kanjarla, S.R. Niezgoda, R.A. Lebensohn, C.N. Tomé, Numerical study of the stress state of a deformation twin in magnesium, Acta. Mater. 84 (2015) 349-358.

[31] P.-A. Juan, S. Berbenni, L. Capolungo, Prediction of internal stresses during growth of first- and second-generation twins in $\mathrm{Mg}$ and $\mathrm{Mg}$ alloys, Acta. Mater. 60 (2012) 476-486.

[32] S. Berbenni, M. Berveiller, T. Richeton, Intra-granular plastic slip heterogeneities: discrete vs. mean field approaches, Int. J. Solids Struct. 45 (2008) 4147-4172.

[33] K. Tanaka, T. Mori, Note on volume integrals of the elastic field around an ellipsoidal inclusion, J. Elast. 2 (1972) 199-200.

[34] U.F. Kocks, C.N. Tomé, H.R. Wenk, Texture and Anisotropy, Cambridge University Press, Cambridge, 1998.

[35] J. Koike, N. Fujiyama, D. Ando, Y. Sutou, Roles of deformation twinning and dislocation slip in the fatigue failure mechanism of AZ31 Mg alloys, Scr. Mater. 63 (2010) 747-750.

[36] S.G. Hong, S.H. Park, C.S. Lee, Enhancing the fatigue property of rolled AZ31 magnesium alloy by controlling 10-12 twinning-detwinning characteristics, J. Mater. Res. 25 (2010) 784-792.

[37] W. Wu, S.Y. Lee, A.M. Paradowsk, Y.F. Gao, P.K. Liaw, Twinning-detwinning behavior during fatigue-crack propagation in a wrought magnesium alloy AZ31B, Mater. Sci. Eng. A 556 (2012) 278-286.

[38] C.H. Caceres, T. Sumitomo, M. Veidt, Pseudoelastic behaviour of cast magnesium Az91 alloy under cyclic loading-unloading, Acta. Mater. 51 (2003) 6211-6218.

[39] C.F. Gu, L.S. Toth, M. Hoffman, Twinning effects in a polycrystalline magnesium alloy under cyclic deformation, Acta. Mater. 62 (2014) 212-224.

[40] M. Berveiller, O. Fassi-Fehri, A. Hihi, The problem of 2 plastic and heterogeneous inclusions in an anisotropic medium, Int. J. Eng. Sci. 25 (1987) 691-709. 